\title{
Shell Model Description of Isotope Shifts in Calcium
}

\author{
E. Caurier ${ }^{1}$, K. Langanke ${ }^{2}$, G. Martínez-Pinedo ${ }^{2,3}$ \\ F. Nowacki ${ }^{4}$, and P. Vogel ${ }^{5}$ \\ ${ }^{1}$ Institut de Recherches Subatomiques, Université Louis Pasteur, F-67037 Strasbourg, France \\ ${ }^{2}$ Institute of Physics and Astronomy, University of Aarhus, DK-8000 Aarhus C, Denmark \\ ${ }^{3}$ Department für Physik und Astronomie, Universität Basel, CH-4056 Basel, Switzerland \\ ${ }^{4}$ Laboratorie de Physique Théorique, Université Louis Pasteur, F-67037 Strasbourg, France \\ ${ }^{5}$ Department of Physics, California Institute of Technology, Pasadena, CA 91125, USA
}

(October 27, 2018)

\begin{abstract}
Isotope shifts in the nuclear charge radius of even and odd calcium isotopes are calculated within the nuclear shell model. The model space includes all configurations of nucleons in the $2 s, 1 d_{3 / 2}, 1 f_{7 / 2}$, and $2 p_{3 / 2}$ orbits. The shell model describes well the energies of the intruder states in $\mathrm{Sc}$ and $\mathrm{Ca}$, as well as the energies of the low-lying $2^{+}$and $3^{-}$states in the even Ca isotopes. The characteristic features of the isotope shifts, the parabolic dependence on $A$ and the prominent odd-even staggering, are well reproduced by the model. These features are related to the partial breakdown of the $Z=20$ shell closure caused by promotion, due to the neutron-proton interaction, of the $d s$ shell protons into the $f p$ shell.
\end{abstract}

21.10.Ft, 21.60.Cs, 21.60.-n

Introduction The appearance of shell gaps associated with magic nucleon numbers is one of the cornerstones in nuclear structure. However, it has become increasingly evident in recent years that these magic numbers, and the corresponding shell closures, might get eroded with increasing neutron excess. To understand the origin of this erosion and to identify whether this trend applies to all magic numbers is one of the great challenges in nuclear structure with, among other things, strong astrophysical implications. A prominent example is the magic neutron number $N=20$ which vanishes in proton-deficient nuclei with $Z \leq 12$ [1]. This erosion of the shell closure has been related to cross-shell proton-neutron interaction which correlates the $2 s$ and $1 d_{3 / 2}$ proton orbitals with the $1 f_{7 / 2}$ and $2 p_{3 / 2}$ neutron orbitals and leads to appreciable deformation [2]. If cross-shell correlations are indeed the mechanism for the shell erosion, then first indications are already visible in the stable calcium isotopes, as we will argue in this Letter. Our argument is based on the understanding and explanation of the nuclear charge radii, $\left\langle r_{c}^{2}\right\rangle$, in the calcium isotopes which by itself have been a challenge for nuclear theory for the last two decades.

Experiments based on optical isotope shifts as well as on muonic atom data (see Ref. [3 for a summary and original references, in particular [1), revealed a characteristic parabolic shape of the isotope shifts with a pronounced odd-even staggering when neutrons fill the $f_{7 / 2}$ orbit and the mass number changes from $A=40$ to $A=48$. Thus, the challenge consists of understanding $(i)$ why ${ }^{48} \mathrm{Ca}$ is not larger than ${ }^{40} \mathrm{Ca}$, (ii) why the maximum of $\left\langle r_{c}^{2}\right\rangle$ is reached in ${ }^{44} \mathrm{Ca}$, (iii) why odd $A$ calcium isotopes are considerably smaller than the average of their even neighbors, and (iv) last but not least, the actual magnitude of the isotope shifts.

There have been numerous attempts to explain these findings. If they are indeed related to the cross-shell proton-neutron correlations, one expects that in order to describe them, one must include higher than $2 \mathrm{p}-2 \mathrm{~h}$ correlations. This is necessary in order to reproduce the oddeven staggering and, at the same time, shell effects related to the neutron shell closures at $N=20$ and 28. This conjecture is supported by the fact that mean field calculations, which usually aim at describing nuclear masses, deformation parameters, and radii over a large region of nuclear masses and charges, cannot account for the details of the calcium isotope shifts. The dependence of $\left\langle r_{c}^{2}\right\rangle$ on $A$ is usually featureless. Some of the approaches, however, are able to account at least for the property $(i)$, i.e., the near equality of $\left\langle r_{c}^{2}\right\rangle$ in ${ }^{40} \mathrm{Ca}$ and ${ }^{48} \mathrm{Ca}$. To this category belong calculations based on the Hartree-Fock method with Skyrme interactions [5], relativistic mean field methods [6], and the extended Thomas-Fermi model with the Strutinsky-integral [7].

An extension of the mean field method with pairing, the so-called local energy-density functional approach [8], can describe most of the features mentioned above by introducing three- and four-body forces via the density dependence of pairing, and adjusting the corresponding free parameters.

That three-body forces can do the job has been recognized long time ago. In [9] the isotope shifts in Ca were quite well described using the configuration mixing within the $\left(f_{7 / 2} d_{3 / 2}\right)^{n}$ shell model space, and introducing an isoscalar particle-hole force whose strength was proportional to the number of valence nucleons. The origin of that dependence and the magnitude of the corresponding coupling constant, however, remained unexplained.

Below we show that the modern shell model can describe the isotope shifts in calcium consistently and without the need for many-body forces or adjustable parameters. The basic mechanism of the $A$ dependence of the charge radius $\left\langle r_{c}^{2}\right\rangle$ remains the same, however, as in Ref. 
[9]. Due to the configuration mixing across the $Z=20$ shell boundary, protons are lifted from the $s d$ to the $f p$ shell, resulting in the increase of $\left\langle r_{c}^{2}\right\rangle$ equal to

$$
\delta r_{c}^{2}(A)=\frac{1}{Z} n_{f p}^{\pi}(A) \cdot b^{2},
$$

where $Z=20, b$ is the oscillator parameter which we assume remains constant for $A=40-48$, and the number of protons in the $f p$ shell, $n_{f p}^{\pi}(A)$, is the calculated quantity. Below we show how it is is calculated and how one can relate it to other manifestations of breaching of the $Z=20$ shell boundary.

Shell model Progress in the application of modern nuclear shell model has been facilitated by the development of numerical codes (the $m$-code ANTOINE and the $J$-coupled code NATHAN [10]) that can be used with relatively large valence spaces. The selection of the appropriate single-particle space, and the corresponding effective interaction (in particular the choice of its uncertain monopole part [1] ) is the most important starting point of any shell model application.

Since here we are interested in the description of calcium isotopes, it is imperative to include states in the vicinity of the $N=Z=20$ shell boundary. Therefore, the chosen valence space consists of the $d_{3 / 2}, s_{1 / 2}, f_{7 / 2}$ and $p_{3 / 2}$ subshells for both protons and neutrons. (Thus ${ }^{28} \mathrm{Si}$ represents the inert core.) This valence space, first used in Ref. [13], has the advantage that the existing codes make it possible to describe all $\mathrm{Ca}$ isotopes without truncation. (The largest dimension, 34,274,564 in the $m$-scheme basis, is encountered for the ground state of ${ }^{43} \mathrm{Ca}$.) The other advantage is the essential absence of the spurious center-of-mass motion (no attempt to suppress it has been made).

For this valence space we start with the two-body matrix elements (TBME) of Ref. [14], which are defined with respect to the ${ }^{16} \mathrm{O}$ core. The single particle energies are now modified to reproduce the ${ }^{29} \mathrm{Si}$ spectrum. The interaction of Ref. [14] was built in blocks $(s d-s d, s d-f p$ and $f p-f p)$, which incorporate the higher-order excitations (core polarization, $2 p 2 h$ excitations etc...). In particular, the $2 p 2 h$ effects consist mainly in pairing renormalizations. As we will include explicitly such mixing, and to avoid double counting, a schematic pairing hamiltonian was subtracted from the interaction. Finally, the cross $s d-f p$ monopoles of Ref. [14] were adjusted to the masses of neutron-rich isotopic chains. They were here retuned to reproduce the gap at ${ }^{40} \mathrm{Ca}$ and the spectra of ${ }^{39} \mathrm{~K}$ and ${ }^{41} \mathrm{Ca}$.

Results As indicated in Eq. (1) above, we assume that the main cause of variation of the charge radius with changing neutron number is the lifting of protons from the $s d$ shell into the larger $f p$ shell. This feature then suggests that there should be a correlation between the isotope shifts and other manifestations of the incomplete $Z=20$ shell closure, e.g. the appearance of low-lying intruder states. In Fig. 11 the experimental excitation energies of the intruder $3 / 2^{+}$states in odd- $A$ Sc isotopes are compared with the calculated ones. The agreement is very satisfactory, and in particular the lowering of these states in ${ }^{43} \mathrm{Sc}$ and ${ }^{45} \mathrm{Sc}$ is properly described.

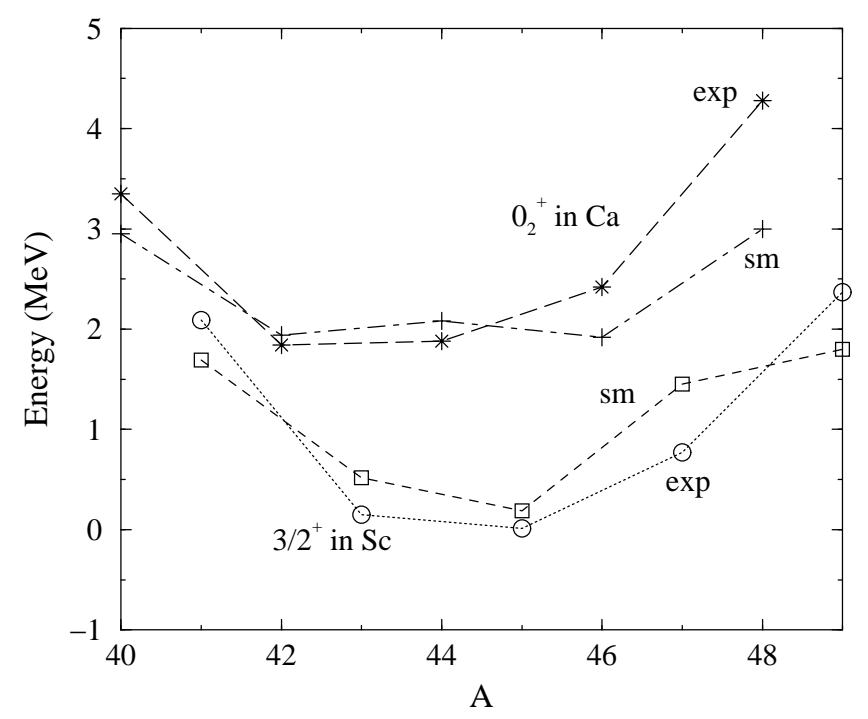

FIG. 1. Excitation energies of intruder states in Sc and in even $\mathrm{Ca}$ isotopes. The experimental (circles) and calculated (squares) energies of the $3 / 2^{+}$states in Sc and the experimental (stars) and calculated (crosses) energies of the excited $0_{2}^{+}$ states in $\mathrm{Ca}$ are shown.

The excited $0_{2}^{+}$states in the even Ca isotopes also belong to the intruder category. Their excitation energies are shown in Fig. 1 as well. Again, the agreement between the experimental and calculated energies is quite good. (The larger discrepancy in ${ }^{48} \mathrm{Ca}$ is presumably caused by the absence of the neutron $f_{5 / 2}$ subshell in the model space.) These $0_{2}^{+}$states are dominantly $4 p-4 h$ states involving both neutron and proton excitations. The parabolic behaviour of both intruder excitations in Fig. 1 suggests that the configuration mixing between the naive shell model configurations and the intruders involving proton excitations from the $s d$ shell will likely be most pronounced near $A=44$ where the corresponding states are lowest.

The occupation numbers for both protons and neutrons, $n_{f p}(A)$, in the ground states of all $A=40-$ $48 \mathrm{Ca}$ isotopes were then evaluated in the full shell model space. It is illustrative to consider, however, how these numbers change when more and more nucleons are allowed to be excited from the naive configuration $(d s)^{6 \pi+6 \nu},(f p)^{(A-40) \nu}$. Varying the number of nucleons, $t$, allowed to be excited from the naive configuration above, from $t=2$ to its maximum value $t=A-28$ we find that the number of holes in the $d s$ shell saturates very slowly. For example, in ${ }^{42} \mathrm{Ca}$ and ${ }^{43} \mathrm{Ca}$ one has to allow $t=6$ and 7 , respectively, just to reach the halfway point of the final $n_{f p}(A)$, and $t=10$ and 11 
to achieve saturation. This means that the "lifting" is a complicated process, involving substantial rearrangement of many nucleons. This can be seen also in the fact that approximately equal numbers of protons and neutrons are lifted from the $d s$ shell. It is therefore difficult to identify a simple cause, or a definite component of the hamiltonian, as the driving force of this effect.

In the full space the quantity $n_{f p}(A)$ is 1.10 in ${ }^{40} \mathrm{Ca}$, reaches its maximum of 1.97 in ${ }^{44} \mathrm{Ca}$, and then decreases again to 0.78 in ${ }^{48} \mathrm{Ca}$.

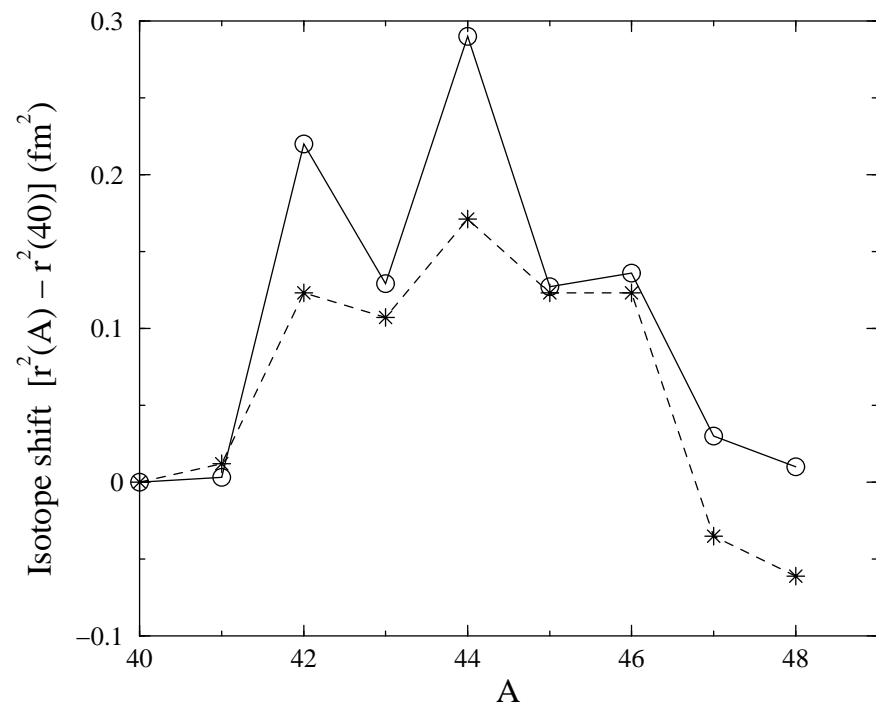

FIG. 2. Isotope shifts in calcium. The experimental data (circles connected by a solid line) and the shell model results, Eq.(1), (stars connected by a dashed line) are shown.

Knowing the quantities $n_{f p}$ one can calculate the isotope shifts from Eq. (1) by subtracting the corresponding $\delta r_{c}^{2}(A=40)$ from $\delta r_{c}^{2}(A)$. (Nucleon form factors contribute negligibly to the isotope shift and thus are neglected.) The results, calculated with a constant $b=1.974 \mathrm{fm}$ as in [9], are shown in Fig. 2 and compared with the experimental values. The trends, i.e. the properties $(i)$ - (iii) are clearly well reproduced, but the magnitude of the calculated shifts is smaller than the experiment suggests.

This cannot be attributed to our choice of the oscillator parameter $b$. Neither it can be cured by replacing the harmonic oscillator wave functions by the WoodsSaxon single-particle wave functions, which changes the isotope shifts shown in Fig. 2 only very little, provided the core $\left\langle r^{2}\right\rangle$ is assumed to be independent of $A$. (Although assuming a very slight increase of $b(A)$, i.e. a gradual increase of the core $\left\langle r^{2}\right\rangle$ from $A=40$ to $A=48$, might "straighten" the curve a bit.) Moreover, choosing the recommended average dependence of $b$ on the mass number $A$ and isospin $T$ 12 $\left(b^{2}=1.07 A^{1 / 3}(1-\right.$ $\left.\left.(2 T / A)^{2}\right) \exp (3.5 / A) \mathrm{fm}^{2}\right)$ would make the radius of ${ }^{48} \mathrm{Ca}$ considerably larger than the radius of ${ }^{40} \mathrm{Ca}$. The $b(A, T)$ dependence, which on average follows [12], clearly is sub- ject to local shell effects. Most likely, the calculated amplitude of the isotope shift might be modified if the so far neglected effects of the filled $d_{5 / 2}$ and empty $f_{5 / 2}$ orbits are added.

Collective states When considering isotope shifts, one also has to take into account the effect of the zero-point motion associated with the surface vibrational modes (see 115 for a systematic approach to this question. There it is stressed that only the low-lying low-multipolarity surface vibrational states contribute significantly). The collective states increase the mean square radius by

$$
\delta r^{2}=\frac{R_{0}^{2}}{4 \pi} \sum_{\lambda} \beta_{\lambda}^{2}
$$

where $R_{0}$ is the equivalent sharp nuclear radius, and $\beta_{\lambda}$ is the vibrational amplitude of the mode $\lambda$, related to the corresponding $B(E \lambda, 0 \rightarrow J=\lambda)$ value. What matters for the isotope shifts, naturally, is not the absolute value of the amplitudes $\beta_{\lambda}$, but their variation with $A$.

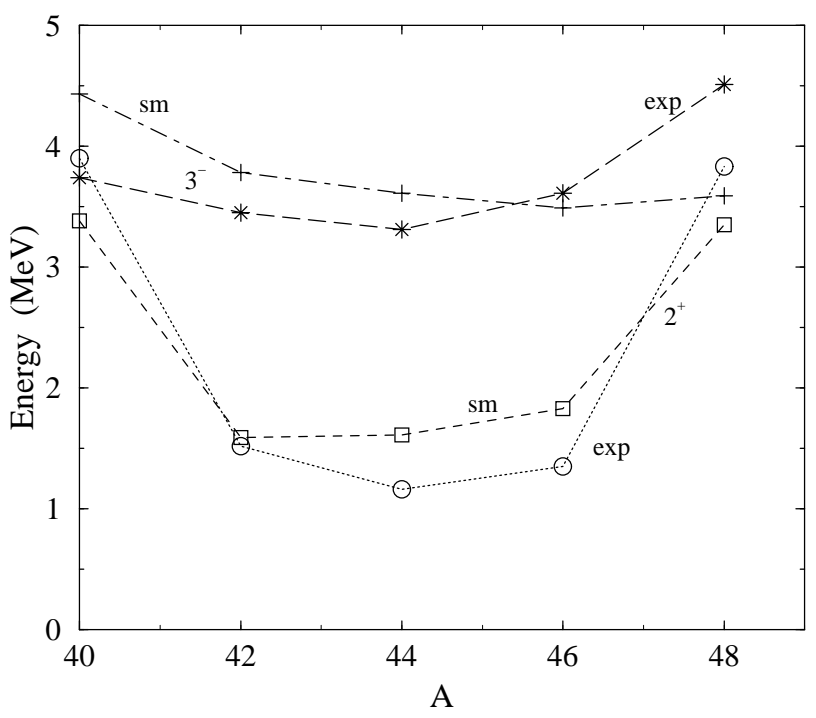

FIG. 3. Excitation energies of the $2^{+}$and $3^{-}$states in the even $\mathrm{Ca}$ isotopes.

Formula (2) was derived as a correction to the mean square radius calculated using the mean-field methods. On the other hand, since the shell model includes all correlations of nucleons in the valence shells, blind use of it would amount to at least partial double counting.

The energies of the lowest lying $2^{+}$and $3^{-}$states are compared to the shell model in Fig. 3. The good agreement there shows not only that the chosen interaction describes this important spectroscopic data well, but that the zero-point motion effect on the nuclear radius is (at least most of it) automatically included.

In addition, in Fig. 4 we compare the experimental and shell model $B(E 2,2 \rightarrow 0)$ values. (The calculations were performed with the usual effective charges $e_{p}=1.5, e_{n}=$ 0.5.) Again, the agreement is quite good, strengthening 


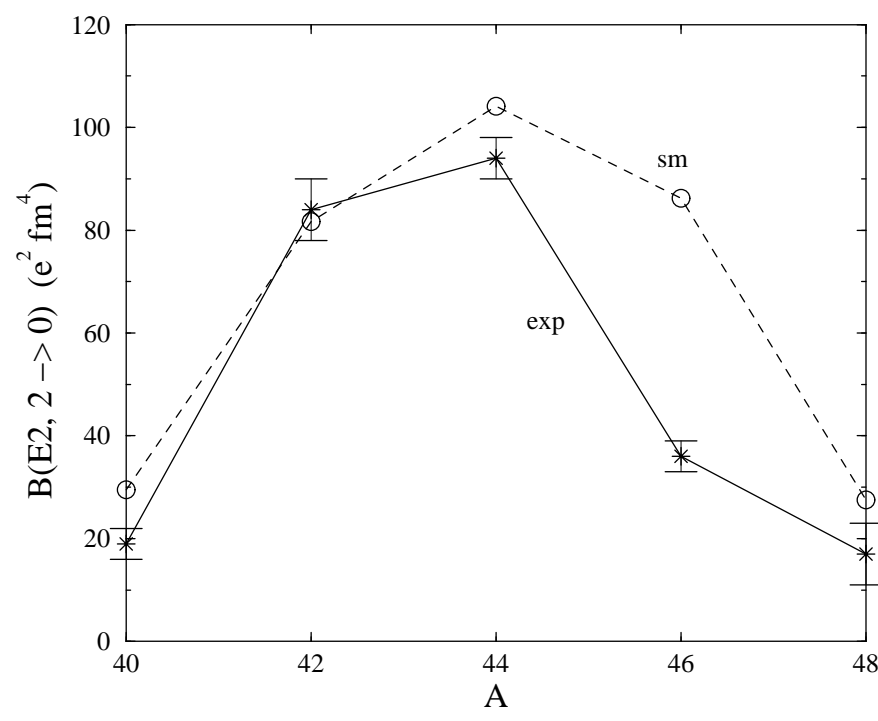

FIG. 4. Comparison of the experimental and shell model $B(E 2,2 \rightarrow 0)$ values in the even Ca isotopes.

our belief that most of the effect of the collective states is already contained in our calculation.

Discussion The present calculation shows that the $Z=20$ and $N=20$ shell boundaries are clearly not absolute, and that substantial configuration mixing involving nucleons from the $d s$ shell is present even in the Ca isotopes.

This finding is in line with the previously recognized and perhaps more dramatic "islands of inversion" related to the configuration mixing involving the $N=20$ shell boundary in neutron rich nuclei (see Ref. 16,17]). Here we are encountering a similar situation, now however in stable nuclei near or at $N=Z$.

Is there any other evidence for the incomplete shell closure in ${ }^{40} \mathrm{Ca}$ ? Indeed, the study of the $(n, p)$ and $(p, n)$ reactions 18 20 on ${ }^{40} \mathrm{Ca}$ revealed that the integrated Gamow-Teller strength $B(G T)=1.6 \pm 0.1$ below $15 \mathrm{MeV}$. Obviously, if the $d s$ shell in ${ }^{40} \mathrm{Ca}$ were really closed, the $B(G T)$ would vanish. Note, that without the full inclusion of the $f_{5 / 2}$ and $d_{5 / 2}$ orbit we can only estimate the total Gamow-Teller strength in ${ }^{40} \mathrm{Ca}$. Without these orbits we obtain the total $B(G T)=0.53$ using the usual quenching of 0.744 [21]. When we allow the $t=1$ (i.e. one particle or one hole) excitations involving the $f_{5 / 2}$ and $d_{5 / 2}$ orbits, we obtain $B(G T)=2.75$ with quenching, in fair agreement with the experiment. Clearly, some of the strength associated with these orbits will be above $15 \mathrm{MeV}$, and should not be counted.

In conclusion, the present calculation shows that the isotope shifts, and the position of the intruder states in $\mathrm{Ca}$ isotopes, can be well described by large scale shell model calculations. The judicious choice of the valence space and the corresponding effective hamiltonian is the key ingredient to this success. Thus, the challenge to the nuclear structure theory, described in the introduction to this work, has been largely met. No new forces or parameters are needed to describe the dependence of the nuclear radius in the odd and even $\mathrm{Ca}$ isotopes on the mass number $A$.

Acknowledgment This work has been partially supported by a grant of the Danish Research Council, Carlsberg Foundation, Sweizerische Nationalfonds, and by the U. S. Department of Energy. F. N. and P. V. thank the Institute of Nuclear Theory, Seattle, where part of the work was done, for hospitality. In addition, E. C. and P. V. thank the Institute of Physics and Astronomy at the University of Aarhus for its hospitality.

[1] T. Motobayashi et al., Phys. Lett. B346, 9 (1995).

[2] E.K. Warburton, J.A. Becker and B.A. Brown, Phys. Rev. C 41, 1147 (1990); N. Fukunishi, T. Otsuka and T. Sebe, Phys. Lett B296, 279 (1992); A. Poves and J. Retamosa, Nucl. Phys. A571, 224 (1994); D.J. Dean et al., Phys. Rev. C 59, 2474 (1999); Y. Utsuno, T. Otsuka, T. Mizusaki, and M. Honma, Phys. Rev. C 60, 4315 (1999).

[3] G. Fricke et al., ADNDT 60, 177 (1995).

[4] C. W. P. Palmer et al., J. Phys. B 25, 2197 (1984).

[5] T. R. Werner et al., Nucl. Phys. A597, 327 (1996). $B(G T)$

[6] G. A. Lalazissis, S. Raman, and P. Ring ADNDT 71, 1 (1999).

[7] Y. Aboussir, J. M. Pearson, A. K. Dutta, and F. Tondeur, Nucl. Phys. A549, 155 (1992).

[8] S. A. Fayans, S. V. Tolokonnikov, E. L. Trykov, and B. Zawisha, Nucl. Phys. A676, 49 (2000).

[9] E. Caurier, A. Poves, and A. Zuker Phys. Lett. 96B, 15 (1980).

[10] E. Caurier, computer code ANTOINE, CRN, Strasbourg, 1989; E. Caurier and F. Nowacki, Acta Physica Polonica B30, 705 (1999).

[11] M. Dufour and A. P. Zuker, Phys. Rev. C 54, 1641 (1996).

[12] J. Duflo and A. P. Zuker, Phys. Rev. C 59, R2347 (1999).

[13] H. Hasper, Phys. Rev. C 19, 1482 (1979).

[14] J. Retamosa, E. Caurier, F. Nowacki and A. Poves, Phys. Rev. C 55, 1266 (1997).

[15] H. Esbensen and G. F. Bertsch, Phys. Rev. C 28, 355 (1983).

[16] E. Caurier, F. Nowacki, A. Poves, and J. Retamosa, Phys. Rev. C 58, 2033 (1998).

[17] E. Caurier, F. Nowacki, and A. Poves, Nucl. Phys. A, to be published.

[18] B. K. Park et al., Phys. Rev. C 45, 1791 (1992).

[19] T. Chittrakarn et al., Phys. Rev. C 34, 80 (1986).

[20] T. N. Taddeucci et al., Phys. Rev. C 28, 2511 (1983).

[21] E. Caurier, A. Poves, and A. P. Zuker, Phys. Rev. Lett. 74, 1517 (1995). 